\title{
Direct detection of exoplanets - science and techniques
}

\author{
Andreas Quirrenbach \\ Leiden Observatory, P.O. Box 9513, NL-2300RA Leiden, The Netherlands
}

\begin{abstract}
The direct detection of light emitted or reflected by extrasolar planets will soon allow studies of the physical properties of their surfaces and atmospheres. This article gives an overview of the scientific perspectives and the techniques that are currently being developed for observations of gas giants and terrestrial planets from the ground and in space.
\end{abstract}

Keywords. astrobiology, atmospheric effects, instrumentation: high angular resolution, instrumentation: interferometers, techniques: high angular resolution, techniques: interferometric, telescopes, stars: imaging, stars: low-mass, brown dwarfs, (stars:) planetary systems

\section{Introduction}

Over the past decade we have witnessed an explosive growth of our knowledge on planetary systems. The discoveries of "Hot Jupiters", of planets with highly eccentric orbits, of multiple systems with orbital resonances, and of transiting planets whose physical properties can be studied, have opened a new and fruitful field of astronomical research.

Further progress in our understanding of planetary systems will depend strongly on the development of new techniques, which can provide detailed information on the planets themselves and on their orbits. Direct detection of exoplanets with coronography and interferometric techniques will enable spectroscopic analyses of their surfaces and atmospheres. Measuring radius, temperature, albedo, atmospheric composition and structure, as well as characterizing surface mineralogy and establishing the presence of surface features, will extend the discipline of physical planetology from the Solar System to other worlds. Understanding climates, the occurrence of water, and habitability will lead to a synoptic view of planetary system diversity. Continued radial-velocity monitoring and precise astrometric observations will yield complementary data on dynamical masses and orbital parameters, interactions and orbital resonances in multiple systems; this new field of planetary system dynamics will provide crucial information needed to test theories of planet formation and migration.

The main technical difficulty of direct planet detection is the very high contrast between planets and their parent stars, combined with their small angular separation. This is illustrated in Fig. 1, which shows the expected star - planet brightness ratios and angular separations for different classes of planets. From this figure it is obvious that the requirements for the detection and characterization of terrestrial planets are much more stringent than those for Jovian planets, which in turn are more difficult to detect than young planets and brown dwarf companions. It is also clear that planets around nearby stars are much more easily accessible than those at larger distance from the Earth. It is thus not difficult to predict that future instruments and facilities will first succeed in detecting and characterizing the nearest Jovian gas giants, and then progress towards smaller planet sizes and towards larger samples. Putative "Earth twins" provide a useful benchmark, because of their inherent appeal both to professional planetologists and to the general public. 


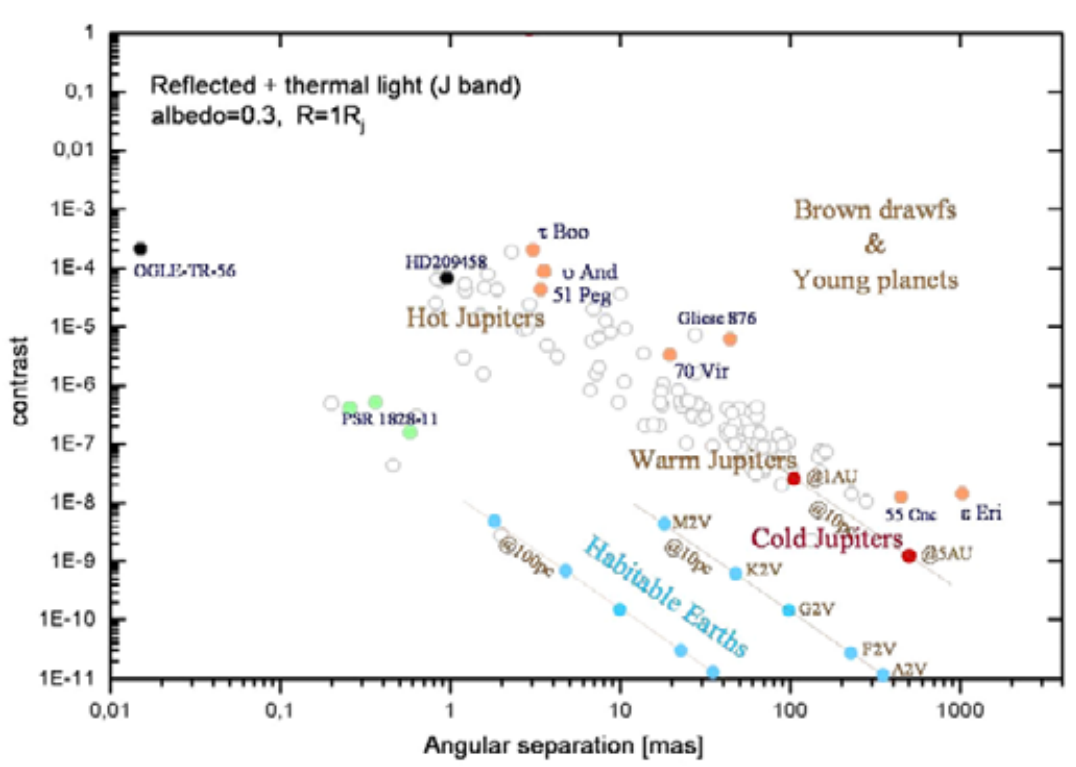

Figure 1. Planet families in the contrast vs. separation plane. The difficulty of detection increases from the top right (low contrast - large separation) to the bottom left (high contrast small separation). The expected positions of known extrasolar planets are indicated, based on models for their atmospheres. Analogs of the Earth in the habitable zones of stars with different spectral types are shown at $10 \mathrm{pc}$ and $100 \mathrm{pc}$.

While our expectations for extrasolar planets are thus shaped by the examples found in our own Solar System, one must also remain prepared for finding the unexpected. Amongst the more exotic possibilities that have recently been discussed are "ocean planets" (Kuchner 2003, Léger et al. 2004). About half of the mass of this hypothetical type of planet would consist of metals and silicates, the other half of water, which would form a thick ice mantle. Such objects could form in the outer parts of planetary systems (beyond the snow line), and migrate inwards. If they reach the habitable zone, the surface ice would melt, and cover the planet with a global ocean. When claims are made that a certain technique can distinguish between different types on planets (based on their mass, brightness, colors, or other physical properties), one must thus keep in mind that possibilities exist for which there is no prototype in the Solar System.

\section{Gas giants}

\subsection{Luminosity evolution and contrast}

The global evolution of isolated giant planets is characterized by secular cooling, which reduces their luminosities by three to four orders of magnitude between ages of a few Myrs and a few Gyrs (e.g., Fig. 7 in Burrows et al. 1997). Stellar illumination slows down the cooling, and adds a reflected light component to the thermal emission. This creates a dichotomy in the preferred detection strategies: young warm planets are most easily detectable through their thermal emission at wavelengths of $\sim 2 \mu \mathrm{m}$ or longer; cool old planets at several AU (i.e., analogs to Jupiter) can probably better be observed in reflected light at visible wavelengths.

The brightness of cool gas giants can be estimated relatively easily by scaling from Jupiter, whose illuminated hemisphere is $\sim 3 \cdot 10^{-9}$ as bright as the Sun at visible wavelengths. (Note that for realistic calculations one has to take phase effects into account; 
at maximum elongation in a circular orbit only half of the visible hemisphere is actually illuminated!) Since the radius of gas giants is nearly independent of mass for a very large mass range, detecting more massive planets in this way is not any easier. The contrast of Jupiter analogs thus looks discouragingly high, but detecting the reflected light of gas giants in $\approx 1 \mathrm{AU}$ orbits (which are $\approx 25$ times brighter) may become possible in the near future (see Sect. 2.4).

The situation is more complicated for young planets, because of the degeneracy of mass $M$ and age $A$ for a given luminosity $L$. Very roughly, $L \propto M^{2} A^{-1}$, which means that uncertainties in the stellar age translate into large error bars on the estimated mass. The situation is even worse for very young planets with ages of only a few Myrs. For example, the companion of the T Tauri star GQ Lupi may have a mass anywhere in the range $1 \ldots 42 M_{\text {Jup }}$, depending on which models are used (Neuhäuser et al. 2005).

\subsection{Transiting Planets}

While strictly speaking observations of planets transiting in front of the disks of their parent stars do not qualify as "direct detections", they can address a number of the issues that usually require the detection of photons emitted or reflected by the planet. Transit data can be used to derive planetary radii. All but one of the known transiting planets are just slightly larger than Jupiter, in agreement with models that include insolation (e.g., Laughlin et al. 2005). The one exception that is larger than expected is HD $209458 \mathrm{~b}$.

Planetary transits also provide special opportunities to perform transmission spectroscopy of their atmospheres. At the wavelengths of atmospheric features the planet blocks more starlight than at continuum wavelengths, thus causing a slightly deeper eclipse. The characteristic fractional coverage of the atmosphere projected against the stellar disk $\delta A / A$ is given by the area of an annulus one atmospheric scale height $H$ thick around the planet, divided by the area of the stellar disk. We therefore have

$$
\left(\frac{\delta A}{A}\right)_{\text {atmos }}=\frac{2 \pi R_{p} H}{\pi R_{*}^{2}}=\frac{2 R_{p}(k T / g \mu)}{R_{*}^{2}},
$$

where $R_{*}$ and $R_{p}$ are the radii of the star and the planet, $T$ and $g$ are the temperature and surface gravity of the planet, and $\mu$ the mean molecular weight of the atmospheric constituents. This signal is $2 H / R_{p}$ smaller than the transit depth. The detection of sodium in the atmosphere of HD $209458 \mathrm{~b}$ with the STIS instrument on HST (Charbonneau et al. 2002) with this technique marks the beginning of studies of extrasolar planetary atmospheres.

The secondary eclipse, when the planet passes behind the star, can be used to determine the thermal emission of the planet by differencing flux measurements out of the eclipse (star + planet) and during the eclipse (star only). This has been achieved for the transiting systems TrES-1 (Charbonneau et al. 2005) and HD 209458 (Deming et al. 2005) with the Spitzer Space Telescope instruments IRAC and MIPS, respectively.

\subsection{High-Contrast Imaging Today}

The angular separations of extrasolar planets from their parent stars are almost always $<1^{\prime \prime}$ (see Fig. 1), within the seeing halo of ground-based observations. This seeing halo can be reduced, but never completely eliminated by adaptive optics. Ground-based highcontrast imaging is therefore always a combination of sophisticated adaptive optics with a differential technique that reduces the effect of the remaining speckle noise. If simultaneous images in two narrow bands closely adjacent in wavelength are subtracted from each other, the speckle noise is reduced drastically (Racine et al. 1999). If the two wavelengths are chosen to bracket a molecular band head that is expected to be strong in 
giant planets and brown dwarfs (such as the methane band head at $1.59 \mu \mathrm{m}$ ), the faint object will stand out in the difference because it is much brighter in one channel than in the other. This simultaneous differential imaging (SDI) technique has been implemented in the NAOS/CONICA instrument at ESO's Very Large Telescope (Lenzen et al. 2005). This system was used by Close et al. (2005) for observations of the young low-mass star AB Dor C, which is 120 times fainter than AB Dor A at a separation of 0 "' 16 - an impressive result, but still far from what is needed for the detection of extrasolar planets.

Systematic searches for faint companions are now being conducted with a number of instruments, but so far none has uncovered a true extrasolar planet. The object that arguably comes closest is the companion to the brown dwarf 2MASSW J1207334-393254, which has an estimated mass of $5 M_{\text {Jup }}$ (Chauvin et al. 2005).

\subsection{The Next Steps on the Ground}

The next step towards higher contrast will require more capable adaptive optics systems, which can provide a high-quality point spread function (Strehl ratio $\gtrsim 80 \%$ ) at the observing wavelength. This enables the use of a coronograph, in which a mask is placed in the focal plane to reject the light from the bright on-axis star. To prevent the light diffracted at this mask from entering the detector, a stop is placed in a second pupil plane, which is formed by suitable re-imaging optics. Wavefront errors compromise the ability of any coronograph to reject the starlight; wavefront control and correction are therefore of paramount importance for coronographs on the ground and in space. A high-performance coronographic system has been built for the $3.6 \mathrm{~m}$ AEOS telescope in Maui, which is equipped with a state-of-the-art high-order adaptive optics system (Oppenheimer et al. 2004).

Several groups are currently designing instruments for $8 \mathrm{~m}$ class telescopes optimized specifically for the detection of giant extrasolar planets. Two parallel studies have been conducted for a Planet Finder instrument at the VLT (Feldt et al. 2003, Mouillet et al. 2004); the two teams have meanwhile been merged. The Planet Finder project includes three differential instruments: a visible-light imaging polarimeter (Gisler et al. 2004), a near-IR integral-field spectrograph, and a near-IR SDI camera. Whereas the first of these instrument concepts is optimized for the detection of cold old planets, the other two will be used for observations of warm young systems. The XAOPI study, which intends to provide a similar capability at the Keck or Gemini observatories, puts more emphasis on advanced adaptive optics components such as a micro-optical (MEMS) deformable mirror (Macintosh et al. 2004).

\subsection{Coronography in Space}

Coronography is much easier in space, where one does not have to contend with the turbulence and dispersion of the atmosphere. Therefore a telescope of modest size $(1.5 \mathrm{~m}$ to $2 \mathrm{~m}$ ), equipped with adaptive optics to correct imperfection of the optics and a high-performance coronograph, would be sufficient for visible-light studies of gas giants (Trauger et al. 2003, Clampin et al. 2004). The maturity of the wavefront correction technology has been demonstrated in laboratory tests, which have achieved a contrast of $1.5 \cdot 10^{-9}$ (Trauger et al. 2004).

\subsection{Planetary Spectroscopy with Interferometry}

Differential phase observations with a near-IR interferometer offer a way to obtain spectra of extrasolar planets. The method makes use of the wavelength dependence of the interferometer phase of the planet/star system, which depends both on the interferometer geometry and on the brightness ratio between the planet and the star (Quirrenbach 2000). 
This brightness ratio varies considerably between molecular bands, where the planet is very dark, and wavelength regions between these bands, where it is relatively bright. In the case of $51 \mathrm{Peg}$ b, which is quite favorable because the planet is close to the star and therefore hot and bright, the expected effect on the interferometer phase is $\sim 0.5 \mathrm{mrad}$ on a $200 \mathrm{~m}$ baseline. This could in principle be measured with a signal-to-noise ratio of $\sim 3000$, but the differential phase is strongly affected by instrumental and atmospheric dispersion. Difficulties in calibrating these effects might prevent the application of the differential phase method to systems with a very high contrast, such as extrasolar planets. A promising alternative is the use of spectrally resolved closure phases, which are immune to many of the systematic and random errors affecting the single-baseline phases.

Joergens \& Quirrenbach (2004) have computed the response of the AMBER instrument at the VLTI to a realistic model of the 51 Peg system, taking into account a theoretical spectrum of the planet as well as the geometry of the VLTI. They have also presented a strategy to determine the geometry of the planetary system and the spectrum of the extrasolar planet from such closure phase observations in two steps, without any prior assumptions on the system geometry or the planet spectrum. First, there is a close relation between the nulls in the closure phase and the nulls in the corresponding singlebaseline phases: every second null of a single-baseline phase is also a null in the closure phase. This means that the nulls in the closure phase do not depend on the spectrum but only on the geometry, so that the geometry of the system can be determined by measuring the nulls in the closure phase at three or more different hour angles. In the second step, the known geometry can then be used to extract the planet spectrum directly from the closure phases. This appears to be the most promising approach to determining the spectra of "Hot Jupiters", which cannot be resolved from their parent stars by single telescopes, but the method could also be applied to other systems.

\section{Terrestrial planets}

\subsection{Biomarkers in terrestrial atmospheres}

Life on Earth has a profound impact on the composition of the atmosphere, producing abundant oxygen, and methane in extreme thermodynamic disequilibrium. The Earth fly-by of the Galileo spacecraft in 1990 demonstrated beautifully that these signatures of life can be detected by remote sensing; in addition the spacecraft detected "a widely distributed surface pigment with a sharp absorption edge in the red part of the visible spectrum" (Sagan et al. 1993); this is the "vegetation red edge" characteristic of land plants. It is plausible to assume that any form of abundant life on an extrasolar planet might transform the planetary atmosphere in a similar way, and thus produce a signature that can be detected spectroscopically (Des Marais et al. 2002).

The mid-infrared spectral region $(\sim 6 \ldots 20 \mu \mathrm{m})$ contains a number of important spectral features that can be used to diagnose the presence of $\mathrm{H}_{2} \mathrm{O}, \mathrm{CO}_{2}$, and $\mathrm{O}_{3}$, which serves as a proxy for $\mathrm{O}_{2}$ (Angel et al. 1986, Léger et al. 1996). Methane also has a strong absorption band in this spectral region, but it would be extremely difficult to detect in an Earth-like atmosphere. In the past, however, before the rise of oxygen, biogenic $\mathrm{CH}_{4}$ may have been more abundant in the Earth's atmosphere by a factor 100 . 1000 (Catling et al. 2001); at this level it would also be detectable and serve as an indicator of bacterial life. One cautionary remark is in order, however: the detection and interpretation of infrared absorption bands could be complicated by the presence of cirrus clouds, which tend to reduce the depth of these features (Smith et al. 1993).

There are of course also many molecular absorption bands in the visible and near-IR spectral ranges. Using the oxygen A band at $7600 \AA$ as a tracer of life was indeed proposed 
much earlier than the mid-IR signatures discussed above (e.g. Owen 1980). Ozone has a broad band near $6000 \AA$, and a very strong band in the UV that is responsible for the short-wavelength cutoff in the transmission of our atmosphere. Water also has very strong absorption bands; the gaps between these bands define the astronomical $J, H, K$, and $L$ bands in the near-IR. In addition, the $\mathrm{CH}_{4}$ and $\mathrm{CO}_{2}$ bands in the $2 \mu \mathrm{m}$ spectral region could serve as a diagnostic of elevated levels of these gases.

Mining the wealth of information potentially available in the visible/near-IR has its own complications, however. A wide range of plausible values of the albedo translates into a corresponding uncertainty in the planetary brightness. There are large differences between the colors and spectra reflected from oceans, snow, deserts, and forested areas. Clouds also cause very marked changes of albedo, UV-visible color, and depth of the oxygen bands (Burrows et al. 1999). It is of course possible to take advantage of these effects with photometric measurements, from which the rotation period, presence of marked surface features, fractional cloud cover, and perhaps seasonal variations of these quantities could be derived (Ford et al. 2001). Again, caution has to be exercised in the interpretation of the data, as for example aerosols can reduce the contrast and therefore suppress diurnal variations (King et al. 1999).

\subsection{The Quest for Habitable Planets}

The search for terrestrial exoplanets is intimately linked to the quest for extraterrestrial life. The emergent discipline of astrobiology seeks to place life as we know it on Earth into a cosmic context (e.g., Chyba \& Hand 2005). Therefore the issue whether or not a terrestrial planet is "habitable", i.e., whether it offers conditions favorable for the genesis and survival of life, is of central importance. Planetary habitability is usually and justifiably — associated with the presence of liquid water, and therefore with a temperate climate. For each type of star, one can thus define a "habitable zone", which is the distance range from the star within which a planet's climate can be stabilized by geological feed-back cycles (e.g., Kasting \& Catling 2003).

It should be emphasized that a planet within the habitable zone is not necessary habitable even if its mass is around $1 M_{\oplus}$; other factors, such as the delivery of water during the late stages of accretion may play an important role (Raymond et al. 2004). This implies that comparative studies of sizeable samples of terrestrial planets have the potential to provide new insights into the processes that created the conditions necessary for the emergence of life on Earth.

Over the past few years, ESA and NASA have therefore formulated rather similar goals for a mission - known as "Darwin" in Europe and as "Terrestrial Planet Finder" (TPF) in the US - aimed at performing detailed studies of Earth-like planets in the habitable zones of $\sim 150$ solar type (spectral types F, G, and K) stars. (For a recent summary of ESA's plans within the Cosmic Vision 2015-25 framework see Turon et al. 2006 and Quirrenbach 2006.) These missions would characterize the orbital and physical properties of all detected planets to assess their habitability, and characterize the atmospheres and search for potential biomarkers among the brightest candidates for Earth-like planets. From these requirements it follows that Darwin / TPF must be able to separate planets in the habitable zone from their parent stars. This translates to an angular resolution of $\sim 35$ mas, corresponding to $0.35 \mathrm{AU}$ at $10 \mathrm{pc}$, or $0.7 \mathrm{AU}$ at $20 \mathrm{pc}$.

\subsection{Space-Based Coronography}

At visible wavelengths, the contrast between the Earth and the Sun is a few times $10^{-10}$. Building a telescope that can give such a dynamic range over a separation of $\sim 35$ mas is 
a daunting task. NASA's TPF-C concept intends to solve this problem with a space telescope equipped with an optimized coronograph and a deformable mirror for suppressing scattered light (Ford et al. 2004). An smaller mission as discussed in Sect. 2.5 could serve as a scientific and technical precursor.

A circular pupil is not necessarily the optimum geometry for TPF-C; an elliptical pupil with an axial ratio of roughly $2: 1$ (e.g., $6 \times 3 \mathrm{~m}$ ) would give sufficient resolution along the major axis, and it could be launched much more easily than a telescope with a $6 \mathrm{~m}$ circular aperture. More exotic pupils may also offer significant advantages. Nisenson \& Papaliolios (2001) present simulations of an apodized square aperture, which gives a very clean point spread function, except in the two directions aligned with the edges. Non-apodized pupils that give extremely dark point spread functions along one axis can also be designed (Kasdin et al. 2003). A non-circular telescope would have to be rotated around the lineof-sight for observations aimed at planet detection; the spectroscopic follow up could be done with the optimum orientation. For any of the suggested geometries, manufacturing an optical system with the required exquisite control of scattered light remains the largest technological hurdle. An overview of the status of the technology development for TPF-C has been provided by Quirrenbach (2005).

\subsection{Mid-Infrared Interferometry}

To achieve the required angular resolution in the mid-infrared an interferometer with baselines of at least $50 \ldots 100 \mathrm{~m}$ is required to separate the planets from their host stars. It appears that mounting each telescope and the beam combiner on a separate spacecraft will be much easier than building a single large structure, and therefore a "free-flyer" approach has been adopted for the European Darwin and American TPF-I projects (Kaltenegger \& Fridlund 2005, Henry et al. 2004).

In an infrared interferometer the starlight can be suppressed by introducing an achromatic $\pi$ phase shift in one arm of the interferometer, so that there is destructive interference for light arriving on-axis (Bracewell 1978). In a single-baseline interferometer one would also have to know the absolute null depth very precisely — otherwise a planet could not be distinguished from a slightly increased contribution from the stellar photons leaking through the "destructive" output of the interferometer. This is almost impossible to achieve in practice as the planet - star contrast is $\sim 10^{-6}$; therefore the configurations proposed for Darwin are based on at least three telescopes forming separate nulling interferometers, whose outputs can be combined with time-variable phases, which produces a modulated signal proportional in strength to the brightness of the planet (e.g., Kaltenegger et al. 2004). A Darwin mission consisting of three $3 \mathrm{~m}$ telescopes and capable of performing a survey of $\sim 150$ stars could be ready for launch around 2015 . The current mission concept foresees a dual Soyuz / Fregat launch to put the four spacecraft into a Lissajous orbit around the L2 Lagrange point. The most challenging technologies that will have to be developed for Darwin are the nulling of mid-infrared light with high precision, and the formation flying of the three telescopes and the beam combiner spacecraft. NASA is also continuing work on an interferometric implementation of TPF (called TPF-I), which may at some point lead to a joint ESA/NASA project.

\subsection{Ground-Based Detection of Terrestrial Planets}

Future extremely large telescopes with diameters $\gtrsim 30 \mathrm{~m}$ will undoubtedly make substantial contributions to studies of giant planets. Whether or not ground-based telescopes with diameters as large as $\sim 100 \mathrm{~m}$ could also be used to detect and characterize terrestrial planets has been the subject of some debate. For planet detection, increasing the telescope diameter $D$ gives two separate advantages: first, the number of photons 
Table 1. Measurement synergies for different techniques aimed at characterizing terrestrial exoplanets (adapted from Beichman et al. 2006). The columns are for space astrometry (e.g., SIM Planet Quest), visible-light spectroscopy (e.g, TPF-C), and mid-infrared spectroscopy (e.g., Darwin, TPF-I). "Meas" indicates a directly measured quantity from a mission; "Est" indicates that a quantity that can be roughly estimated from a single mission; and "Coop" indicates a quantity that is best determined cooperatively using data from several missions.

Astrometry Visible Mid-IR

\begin{tabular}{lccc}
\hline Orbital Parameters & & & \\
$\quad$ Stable orbit in habitable zone & Meas & Meas & Meas \\
Characteristics for Habitability & & & \\
$\quad$ Planet temperature & Est & Est & Meas \\
Temperature variability due to eccentricity & Meas & Meas & Meas \\
Planet radius & Coop & Coop & Meas \\
Planet albedo & Coop & Coop & Coop \\
Planet mass & Meas & Est & Est \\
Surface gravity & Coop & Coop & Coop \\
Atmospheric and surface composition & Coop & Meas & Meas \\
Time variability of composition & & Meas & Meas \\
Presence of water & & Meas & Meas \\
Planetary System Characteristics & & & \\
Influence of other planets, orbit coplanarity & Meas & Est & Est \\
Comets, asteroids, and zodiacal dust & & Meas & Meas \\
Indicators of Life & & Meas & Meas \\
Atmospheric biomarkers & & Meas &
\end{tabular}

collected from the planet increases with $D^{2}$; second, the area subtended by the point spread function decreases with $D^{-2}$ (provided that good adaptive optics correction and differential speckle suppression is achieved). Both advantages combine to a scaling of the observing time with $D^{-4}$. Telescope size is therefore a very strong advantage for high-contrast imaging.

Calculations of the fundamental limits set by photon and speckle noise indicate that Earth analogs at $10 \mathrm{pc}$ could be detected by a $100 \mathrm{~m}$ telescope with integration times of order $12 \mathrm{hrs}$ (Angel 2003, Chelli 2005). On the other hand, it is also clear that the technical challenges are formidable. The light diffracted at gaps between the segments of the primary (and possibly also secondary) mirror must be reduced by specially designed coronographs (Sivaramakrishnan \& Yaitskova 2005), and the segments themselves must be co-phased to a small fraction of the wavelength (Lardière et al. 2004). The requirements on wavefront quality in other parts of the telescope and instrument are also of the order of a few $\mathrm{nm}$. It is presently not clear that manufacturing and controlling optics with that quality and stability will be feasible and affordable. Furthermore, fundamental photon noise constraints impose limits on the instantaneous contrast that can be achieved for stars of a given brightness (Stapelfeldt, these proceedings). Therefore the residual speckles at $\sim 100$ mas will on average be $\sim 1,000$ times brighter than a terrestrial planet, and one has to rely on averaging over many independent realizations of the speckle pattern to detect the planet. Whether or not that will be possible remains an open issue.

\subsection{Synoptic Information for Planet Characterization}

Whether information is gathered in the mid-IR or in the visible/near-IR, careful modeling of the planetary atmosphere is required to rule out an abiotic origin of $\mathrm{O}_{2}, \mathrm{CH}_{4}$, or any other presumed indicator of life (e.g. Schindler \& Kasting 2000). One has to keep 
in mind that the biosphere, atmosphere, hydrosphere, and lithosphere interact in many complicated ways. Even reconstructing the processes that lead to the rise of oxygen in our own atmosphere remains a challenging task, in spite of the wealth of available data (Kasting 2001). It will certainly be much more difficult to interpret the glimpses of atmospheric chemistry that we may be able to obtain by remote sensing of extrasolar terrestrial planets. It is therefore important to develop complementary observing capabilities, which include dynamical mass measurements in addition to spectroscopy in multiple wavebands (see Tab. 1). As pointed out by Beichman et al. (2006), a synoptic approach is needed to resolve ambiguities in the information from one mission alone, and to enable robust modeling of planetary properties.

\section{Acknowledgements}

This paper is based partly on presentations given at the IAU Colloquium 200 "Direct Imaging of Exoplanets: Science \& Techniques", which took place in Villefranche-surmer in October 2005. I thank the participants of that conference for many stimulating discussions. Olivier Lardière kindly supplied Fig. 1.

\section{References}

Angel, J.P.R., Cheng, A.Y.S. \& Woolf, N.J. 1986, Nature 322, 341-343

Angel, R. 2003, in: Fridlund, M. \& Henning, T. (eds.) Towards other Earths - DARWIN/TPF and the search for extrasolar terrestrial planets. ESA SP-539, pp. 221-230

Beichman, C.A., Fridlund, M., Traub, W.A., Stapelfeldt, K.R., Quirrenbach, A. \& Seager, S. 2006, in: Protostars and planets $V$, in press

Bracewell, R.N. 1978, Nature 274, 780-81

Burrows, A., Marley, M., Hubbard, W.B., Lunine, J.I., Guillot, T., et al. 1997, ApJ 491, 856-875

Burrows, J.P., Weber, M., Buchwitz, M., Rozanov, V., Ladstätter-Weißenmayer, A., et al. 1999, JAtmSci 56, 151-175

Catling, D.C., Zahnle, K.J. \& McKay, C. 2001, Science 293, 839-843

Charbonneau, D., Brown, T.M., Noyes, R.W. \& Gilliland, R.L. 2002, ApJ 568, 377-384

Charbonneau, D., Allen, L.E., Megeath, S.T., Torres, G., Alonso, R., et al. 2005, ApJ 626, 523-529

Chauvin, G., Lagrange, A.M., Dumas, C., Zuckerman, B., Mouillet, D., et al. 2005, A $8 A 438$, L25-L28

Chelli, A. 2005, A\&A 441, 1205-1210

Chyba, C.F. \& Hand, K.P. 2005, ARAA 43, 31-74

Clampin, M., Melnick, G., Lyon, R., Ford, H., Angel, J., et al. 2004, in: J.C. Mather (ed.), Optical, infrared, and millimeter space telescopes SPIE 5487, pp. 1538-1544

Close, L.M., Lenzen, R., Guirado, J.C., Nielsen, E.L., Mamajek, E.E., et al. 2005, Nature 433, $286-288$

Deming, D., Seager, S., Richardson, L.J. \& Harrington, J. 2005, Nature 434, 740-743

Des Marais, D.J., Harwit, M.O., Jucks, K.W., Kasting, J.F., Lin, D.N.C., et al. 2002, Astrobiology 2, 153-181

Feldt, M., Hippler, S., Henning, T., Gratton, R., Turatto, M., Waters, R. \& Quirrenbach, A. 2003, in: D. Deming \& S. Seager (eds.), Scientific frontiers in research on extrasolar planets. ASP Conf. Ser. Vol. 294, pp. 569-572

Ford, E.B., Seager, S. \& Turner, E.L. 2001, Nature 412, 885-887

Ford, V.G., Lisman, P.D., Shaklan, S.B., Trauger, J.T., Ho, T., et al. 2004, in: J.C. Mather (ed.) Optical, infrared, and millimeter space telescopes SPIE 5487, pp. 1274-1283

Gisler, D., Schmid, H.M., Thalmann, C., Povel, H.P., Stenflo, J.O., et al. 2004, in: A.F.M. Moorwood \& M. Iye (eds.) Ground-based instrumentation for astronomy SPIE 5492, pp. $463-474$

Henry, C., Lay, O., Aung, M., Gunter, S., Dubovitsky, S. \& Blackwood, G. 2004, in: W.A. Traub (ed.) New frontiers in stellar interferometry SPIE 5491, pp. 265-274 
Joergens, V. \& Quirrenbach, A. 2004, in: W.A. Traub (ed.) New frontiers in stellar interferometry SPIE 5491, pp. 551-559

Kaltenegger, L. \& Fridlund, M. 2005, AdSpR 36, 1114-1122

Kaltenegger, L., Karlsson, A. \& Neger, T. 2004, AdSpR 34, 618-624

Kasdin, N.J., Vanderbei, R.J., Spergel, D.N. \& Littman, M.G. 2003, ApJ 582, 1147-1161

Kasting, J.F. 2001, Science 293, 819-820

Kasting, J.F. \& Catling, D. 2003, ARAA 41, 429-463

King, M.D., Kaufman, Y.J., Tanré, D. \& Nakajima, T. 1999, BullAmMetSoc 80, 2229-2260

Kuchner, M.J. 2003, ApJ 596, L105-L108

Lardière, O., Carbillet, M., Riccardi, A. \& Salinari, P. 2004, in: D. Bonaccini, B.L. Ellerbroek \& R. Ragazzoni (eds.) Advancements in adaptive optics SPIE 5490, pp. 516-526

Laughlin, G., Wolf, A., Vanmunster, T., Bodenheimer, P., Fischer, D., et al. 2005, ApJ 621, 1072-1078

Léger, A., Mariotti, J.M., Mennesson, B., Ollivier, M., Puget, J.L., et al. 1996, Icarus 123, $249-255$

Léger, A., Selsis, F., Sotin, C., Guillot, T., Despois, D., et al. 2004, Icarus 169, 499-504

Lenzen, R., Close, L., Brandner, W., Biller, B. \& Hartung, M. 2005, in: A.F.M. Moorwood \& M. Iye (eds.) Ground-based instrumentation for astronomy SPIE 5492, pp. 970-977

Macintosh, B., Bauman, B., Wilhelmsen Evans, J., Graham, J., Lockwood,C., et al. 2004, in: D. Bonaccini, B.L. Ellerbroek \& R. Ragazzoni (eds.) Advancements in adaptive optics SPIE 5490, pp. 359-369

Mouillet, D., Lagrange, A.M., Beuzit, J.L., Moutou, C., Saisse, M., et al. 2004, in: J.P. Beaulieu, A. Lecavelier des Etangs \& C. Terquem (eds.) Extrasolar Planets: Today and Tomorrow ASP Conf. Ser. 321, pp. 39-46

Neuhäuser, R., Guenther, E.W., Wuchterl, G., Mugrauer, M., Bedalov, A. \& Hauschildt, P.H. 2005, A\& A 435, L13-L16

Nisenson, P. \& Papaliolios, C. 2001, ApJ 548, L201-L205

Oppenheimer, B., Digby, A., Newburgh, L., Brenner, D., Shara, M., et al. 2004) in: D. Bonaccini, B.L. Ellerbroek \& R. Ragazzoni (eds.) Advancements in adaptive optics SPIE 5490, pp. $433-442$

Owen, T. 1980, in: M.D. Papagiannis (ed.) Strategies for the search for life in the Universe pp. $177-185$

Quirrenbach, A. 2000, in: J. Bergeron \& A. Renzini (eds.) From extrasolar planets to cosmology: the VLT opening symposium pp. 462-467

Quirrenbach, A. 2005, Coronographic methods for the detection of terrestrial planets ESA WPP245 , astro-ph/0502254

Quirrenbach, A. 2006, in: F. Favata \& A. Gimenez (eds.) ESLAB Symposium 2005: Trends in space science and Cosmic Vision 2020 in press

Racine, R., Walker, G.A.H., Nadeau, D., Doyon, R. \& Marois, C. 1999, PASP 111, 587-594

Raymond, S.N., Quinn, T. \& Lunine, J.I. 2004, Icarus 168, 1-17

Sagan, C., Thompson, W.R., Carlson, R., Gurnett, D. \& Hord, C. 1993, Nature 365, 715-721

Schindler, T.L. \& Kasting, J.F. 2000, Icarus 145, 262-271

Sivaramakrishnan, A. \& Yaitskova, N. 2005, ApJ 626, L65-L68

Smith, W.L., Ma, X.L., Ackerman, S.A., Revercomb, H.E. \& Knuteson, R.O. 1993, JAtmSci 50, $1708-1720$

Trauger, J.T., Burrows, C., Gordon, B., Green, J.J., Lowman, A.E., et al. 2004, in: J.C. Mather (ed.) Optical, infrared, and millimeter space telescopes SPIE 5487, pp. 1330-1336

Trauger, J.T., Hull, T., Stapelfeldt, K., Backman, D., Bagwell, R.B., et al. 2003, in: J.C. Blades \& O.H.W. Siegmund (eds.) Future EUV/UV and visible space astrophysics missions and instrumentation SPIE 4854, pp. 116-128

Turon, C., Done, C., Quirrenbach, A., Schneider, P., Aerts, C., et al. 2006, in: F. Favata \& A. Gimenez (eds.) ESLAB Symposium 2005: Trends in space science and Cosmic Vision 2020 in press 